\title{
CIPÓ-CRAVO (TYNNANTHUS FASCICULATUS MIERS - BIGNONIACEAE): ESTUDO FITOQUÍMICO E TOXICOLÓGICO ENVOLVENDO ARTEMIA SALINA
}

\author{
Stem herb (Tynnanthus fasciculatus MIERS-Bignoniaceae): \\ phytochemical and toxicological study involving Artemia
}

\author{
Camilo A. de Carvalho ${ }^{1,2}$; Sérgio L. P. Matta ${ }^{1,3}$; Fabiana C. S. A. Melo ${ }^{1,4}$; \\ Daniel C. F. Andrade ${ }^{5,6}$ Leandro M. de Carvalho $^{7,8}$; Paulo C. do Nascimento ${ }^{7,8}$; \\ Marcelo B. da Silva ${ }^{9,10}$; Marcelo B. da Rosa*11,12; \\ 1 Departamento de Biologia Geral, Universidade Federal de Viçosa, 36570-000, Viçosa-MG, Brasil. \\ ${ }^{2}$ Mestrando em Biologia Celular e Estrutural \\ ${ }^{3}$ Pesquisador do Laboratório de Biologia Celular e Estrutural \\ ${ }^{4}$ Doutora em Biologia Celular e Estrutural \\ ${ }^{5}$ Centro de Oncologia do Leste de Minas - Oncoleste, 35022-250, Gov. Valadares-MG, Brasil. \\ ${ }^{6}$ Farmacêutico Responsável \\ ${ }^{7}$ Departamento de Química, Universidade Federal de Santa Maria, 97110-970, Santa Maria-RS, Brasil. \\ ${ }^{8}$ Professor de Química Analítica \\ ${ }^{9}$ Departamento de Ciências da Saúde, Biológicas e Agrária, Universidade Federal do Espírito Santo - São \\ Mateus, 29931-220, Espírito Santo-ES, Brasil. \\ ${ }^{10}$ Pesquisador do Laboratório de Fitopatologia \\ ${ }^{11}$ Universidade Federal do Pampa, Campus Bagé, 96412-420, Bagé-RS, Brasil. \\ ${ }^{12}$ Professor de Química
}

*Autor para correspondência: marcelo.rosa@unipampa.edu.br

Recebido em 07/02/2009 - Aceito em 20/02/2009

RESUMO: A Tynnanthus fasciculatus MIERS, conhecida na medicina popular como cipó-cravo, tem sido usada como estimulante, anti-helmíntica, afrodisíaca e no tratamento de problemas estomacais. Neste trabalho, uma prospecção fitoquímica visando identificar classes de compostos presentes no extrato aquoso, etanólico e em diclorometano do cipó-cravo foi realizada. Além disso, a $\mathrm{DL}_{50}$ do extrato aquoso da planta foi obtida com base em ensaios toxicológicos utilizando Artemia salina como bioindicador. Os resultados da prospecção fitoquímica indicaram a presença simultânea de taninos, flavonóides e heterosídeos cardiotônicos para os três extratos estudados. $O$ extrato aquoso apresentou $\mathrm{DL}_{50}=43,4 \mathrm{mg} \mathrm{L}^{-1}$, indicando uma alta toxicidade quando comparado a dados da literatura. A toxicidade acredita-se estar vinculada a presença de taninos. Portanto, este trabalho destaca, entre outros aspectos, o risco de exposição do usuário da planta na medicina popular.

PALAVRAS-CHAVES: Tynnanthus fasciculatus, toxicidade, Artemia, espectrofotometria.

ABSTRACT: The Tynnanthus fasciculatus MIERS, is known for Stem herb in the Brazilian phytomedicine and has been used as stimulant, anti-helminthic, aphrodisiac and in the treatment of stomach problems. In this work, a phytochemical study aiming to identify the principal compounds presents in ethanolic, aqueous and dichloromethane Tynnanthus extracts was performed. Besides, $\mathrm{DL}_{50}$ toxicological tests using Artemia as bioindicator were conduced to obtain information about the plant toxicity. The results of the phytochemical prospection indicated a simultaneous presence of tannins, flavonoids and cardiotonic heterosides for all extracts. The aqueous extract presented a $L D_{50}=43.4 \mathrm{mg} \mathrm{L}^{-1}$ indicating a high toxicity when compared with literature data. The toxicity observed for can be correlated with the presence of tannins. Therefore, this work points the risks of the Tynanthus users in the Brazilian phytomedicine. 
KEYWORDS: Tynnanthus fasciculatus, toxicicity, Artemia, spectrophotometry.

\section{INTRODUÇÃO}

A família Bignoniaceae é constituída por 113 gêneros e 800 espécies de plantas arbustivas, arbóreas e trepadeiras. As espécies deste táxon encontram-se distribuídas nas regiões tropicais de todo o mundo, sendo de ocorrência freqüente no continente americano, cujos jacarandás (Jacaranda brasiliana) e Ipês amarelo e roxo (Tabebuia alba e T. avellanedae) são os exemplos mais representativos da família. Plantas destas espécies são muito utilizadas na construção civil, carpintaria e construção de instrumentos musicais devido à natureza rígida da madeira. Além disso, no planejamento urbano é também utilizada como planta ornamental, devido as suas belas florações, sendo o Ipê o exemplo mais conhecido no paisagismo urbano (PAULETTI et al., 2003).

No Brasil, plantas desta família ocorrem desde a Amazônia até o Rio Grande do Sul, não possuindo um habitat único, podendo ser encontradas nos cerrados, Mata Atlântica e região amazônica (LORENZI, 1998).

A espécie Tynnanthus fasciculatus MIERS (Figura 1), pertencente à família Bignoniaceae, é conhecida popularmente como cipó-cravo por possuir aroma semelhante ao do Cravo-da-Índia. Tradicionalmente é utilizada como estimulante geral, anti-helmíntico e adotada popularmente como estimulante e afrodisíaca além de ser utilizada há séculos como medicamento caseiro para combater má digestão e dores no estômago (BURGO et al., 2006; MELO, 2007). Ensaios farmacológicos têm evidenciado uma atividade analgésica do extrato das folhas em camundongos, bem como atividade antioxidante.

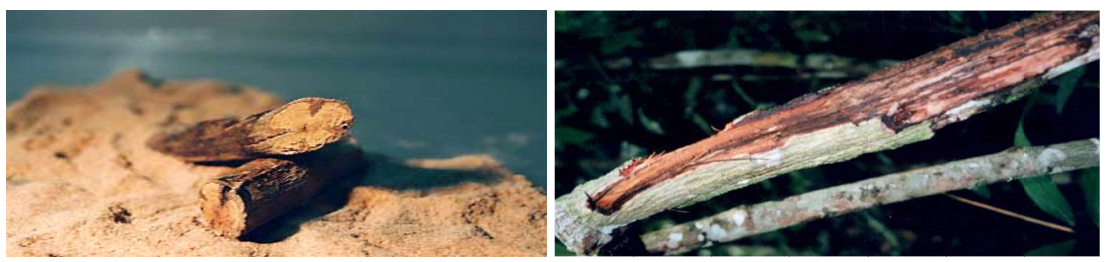

Figura 1. Tynnanthus fasciculatus MIERS (Bignoniaceae). (Fonte: MELO, 2007).

Há registros na literatura de que a Tynanthus tem feito parte de formulações de fitofármacos desde 1937, onde um dos principais compostos encontrados no óleo essencial desta planta é o eugenol e responsável pela atividade anti Claudosporium herbarum, salientando assim seu potencial uso no controle biológico de fungos (BURGO et al., 2006).

Ao longo dos últimos anos, a ocorrência de infecções fúngicas humanas tem apresentando um aumento expressivo, sendo as dermatomicoses as principais infecções responsáveis por esse aumento. Vários fatores estão relacionados ao crescimento dessas infecções fúngicas, entre eles: o melhor diagnóstico laboratorial e clínico, o aumento da sobrevida de pacientes com doenças imunossupressoras e o emprego de medicamentos imunossupressores utilizados às vezes de forma abusiva, permitindo a instalação de microorganismos convencionalmente saprófitos (FENNER et al., 2006). Os fungos responsáveis por essas infecções são fungos patógenos (Epidermophyton, Microsporum, Trichophyton, Paracoccidioides, Histoplasma) ou patógenos oportunistas (Candida albicans, Cryptococcus neoformans). O tratamento das micoses humanas não é sempre efetivo, pois os fármacos antifúngicos disponíveis produzem recorrência ou causam resistência, além de apresentarem certa toxicidade. Por esta razão, há uma busca contínua de novos fármacos antifúngicos mais potentes e sobretudo, mais seguros que os existentes. Além das infecções em humanos, os animais também podem ser atacados esporadicamente por fungos muito difíceis de serem combatidos. Além disso, as infecções fúngicas em plantas por espécies de Rhizoctonia, Phytophtora, Sclerotinia, Fusarium, Phomopsis, Colletotrichum, Diaphorte, Macrophomina, Cercospora, representam perdas incalculáveis para a produção agrícola (FENNER et al., 2006).

Portanto, a busca de novos fármacos antifúngicos a partir de plantas da flora latino-americana, baseada principalmente no seu uso etnofarmacológico assume papel importante neste contexto (FENNER et al., 2006). Sendo assim, justifica-se que há uma grande necessidade de estudos mais detalhados de cada espécie vegetal, a fim de se aumentarem as possibilidades de encontrar novas fontes e/ou alternativas para o tratamento antimicrobiano.

Os compostos produzidos pelos vegetais podem ser reunidos genericamente em dois grupos: os metabólitos primários, tais como carboidratos, aminoácidos e lipídeos e os secundários que são compostos elaborados a partir da síntese dos metabólitos primários, tais como compostos fenólicos, terpenóides, óleos essenciais e alcalóides entre outros (SIMÕES et al., 1999). Esses compostos secundários é que são, em sua maioria, responsáveis pelos efeitos medicinais, ou tóxicos das plantas, além de apresentarem grande importância ecológica, pois atuam na atração de polinizadores e na ativação da defesa química da planta contra estresse ambiental (BALADRIN et al., 1985; DI STASI, 1995).

$\mathrm{Na}$ rotina laboratorial de produtos naturais, diversos ensaios biológicos têm sido inseridos, além do isolamento, purificação e elucidação estrutural dos diversos compostos bioativos (SIQUEIRA et al., 1998). O teste 
frente à Artemia salina (TAS) tem demonstrado boa aceitabilidade decorrente da sua alta sensibilidade, baixo custo, rapidez e ser de fácil manuseio (CAVALCANTE et al., 2000). A Artemia sp. é uma espécie de microcrustáceo da ordem Anostraca, encontrado em águas salgadas (CALOW, 1993). É utilizada como alimento vivo para peixes, sendo seus ovos facilmente encontrados em lojas de aquaristas. Essa espécie marinha tem sido utilizada em experimentos laboratoriais como um bioindicador, sendo o seu grau de tolerância em relação a um fator ambiental reduzido e específico, de modo que apresenta uma resposta nítida frente a pequenas variações na qualidade do ambiente (ABEL, 1989). A letalidade desse organismo tem sido utilizada para identificação de repostas biológicas, onde as variáveis como a morte ou vida são as únicas envolvidas (MEYER et al., 1982). O ensaio de toxicidade com Artemia salina consiste em avaliar a toxicidade aguda do extrato e por isso é fator determinante em bioensaios preliminares, onde o estudo de compostos com potencial atividade biológica é investigado (COLEGATE, 1993).

Assim, o controle de qualidade de fitoterápicos é imprescindível no meio comercial, pois muitas espécies vegetais são vendidas sem quaisquer garantia de qualidade, favorecendo, desde a venda de espécies falsificadas, até o armazenamento inadequado durante a sua comercialização (CARVALHO et al., 2008). Neste estudo, a prospecção fitoquímica foi utilizada no monitoramento de algumas classes de compostos globais constituintes do cipó de Tynnanthus fasciculatus MIERS, bem como na determinação de sua toxicidade frente à Artemia salina.

\section{MATERIAIS E MÉTODOS}

\section{Material botânico}

A coleta do cipó-cravo (T. fasciculatus) foi realizada em janeiro de 2006 junto ao Setor de Dendrologia do Departamento de Engenharia Florestal da Universidade Federal de Viçosa (UFV), Minas Gerais, Brasil. O material coletado foi identificado e autenticado por comparação com espécies do Horto Botânico da UFV, onde a espécie testemunho foi depositada (exsicata $n^{\circ}$ 30.074). O caule de Tynnanthus fasciculatus MIERS foi seco e posteriormente triturado usando-se moinho de martelos (Marconi ${ }^{\circledR}$ - Modelo 340) no laboratório de Ciências Agronômicas da Universidade Vale do Rio Doce (UNIVALE/MG).

\section{Preparação dos extratos}

O triturado foi submetido à extração por ultrasom (Unique ${ }^{\circledR}$ - MaxiClean 1400) durante 60 minutos, com $\mathrm{CH}_{2} \mathrm{Cl}_{2}$ (diclorometano) P.A, $\mathrm{CH}_{3} \mathrm{CH}_{2} \mathrm{OH}$ (álcool etílico) P.A e $\mathrm{H}_{2} \mathrm{O}$ (água). A água utilizada no preparo das soluções e limpeza das vidrarias foi tratada por filtro de osmose reversa $\left(\right.$ Permution $\left.^{\circledR}\right)$. Todas as soluções foram preparadas na concentração de $10 \%(\mathrm{~m} / \mathrm{v}$ - massa de planta seca triturada/volume de solvente). Após este período o extrato concentrado foi filtrado a vácuo (porosidade: $0,2 \mu \mathrm{m}$ ) em funil de Büchner. $\mathrm{O}$ extrato foi elaborado conforme CARVALHO et al., 2008.

\section{Prospecção fitoquímica}

Os extratos aquoso, etanólico e em diclorometano foram submetidos a uma série de reações de caracterização, entre elas: açúcares redutores, compostos fenólicos, naftoquinonas, caracterização de flavonóides, taninos, cumarinas, triterpenos e esteróides, identificação de heterosídeos cardiotônicos e caracterização de saponinas, tendo sido realizadas em triplicata. Os testes se baseiam em reações cromáticas e de precipitação conforme descrito por SIMÕES et al., 1999.

\section{Ensaios de toxicidade - Artemia Salina.}

Utilizou-se a metodologia de MEYER (1982) adaptada por RUIZ et al., (2005). Preparou-se uma solução salina (com sal marinho) na concentração $3,5 \%(\mathrm{~m} / \mathrm{v})$. O pH foi ajustado entre 8,0-9,0 adicionando-se gotas de uma solução $0,1 \mathrm{M} \mathrm{NaOH}\left(\right.$ Merck $^{\circledR}$ ). Esta solução foi utilizada para eclosão dos ovos de Artemia salina e no preparo das outras diluições. Os ovos foram colocados para eclodir na solução salina, por 48 horas, com aeração constante e temperatura controlada de $25^{\circ} \mathrm{C}$.

Cerca de 10 larvas de Artemia salina foram transferidas para tubos de ensaio contendo a solução salina e amostras a serem testadas, nas seguintes concentrações do extrato: $0,1,1,10,50$ e $100 \mathrm{mg} \mathrm{L}^{-1}$. A contagem dos animais mortos e vivos foi realizada após 24h. A morte do microcrustáceo é evidenciada pela sedimentação do crustáceo. Por se tratar de um crustáceo ativo em água salina, a falta de movimento e sedimentação são os indicadores de morte do mesmo.

O teste foi acompanhado de controle negativo, somente com água salina, e positivo com uma solução $0,33 \mathrm{mM}$ de $\mathrm{K}_{2} \mathrm{Cr}_{2} \mathrm{O}_{7}$ (Dicromato de potássio). $A \mathrm{DL}_{50}$ foi estimada a partir da regressão linear obtida da correlação entre a porcentagem de indivíduos mortos e a concentração do extrato. 


\section{RESULTADOS E DISCUSSÃo}

Os estudos fitoquímicos compreendem as etapas de isolamento e identificação dos constituintes majoritários mais importantes das plantas, principalmente de substâncias originárias do metabolismo secundário, responsáveis, ou não, pela ação biológica desejada.

O conhecimento da constituição química das plantas aplicado na medicina popular envolve o estudo de interações do organismo com os efeitos das inúmeras classes de compostos e moléculas que podem existir numa única planta. Esse conhecimento permite identificar a espécie vegetal, conjuntamente com ensaios de atividade biológica, de modo que se pode melhor caracterizar as frações ou substâncias bioativas. Ressalta-se ainda a importância para o desenvolvimento de fitoterápicos o estabelecimento de marcadores químicos, que são indispensáveis para o planejamento e monitoramento das ações de transformações tecnológicas aliado a estudos de estabilidade dos produtos intermediários e finais (TOLEDO et al., 2003).

No que diz respeito à prospecção fitoquímica do cipó-cravo, os resultados da identificação das classes de compostos são apresentados na Tabela 1. Os resultados indicam a presença de metabólitos secundários que podem estar relacionado à sua ação no tratamento de diversos distúrbios.

Tendo em vista o resgate da biodiversidade brasileira e identificação das potencialidades das plantas e sua possível ação medicinal, alguns metabólitos secundários são apontados neste estudo como ponto de partida para este enfoque, sendo apresentados na Tabela 2. Através dessas informações direcionam-se os estudos a fim de se especificar ainda mais o conhecimento sobre a espécie, uma vez que os dados sobre a planta ainda são modestos, quando comparados as suas potencialidades, o que possibilita a descoberta de novos princípios ativos, por exemplo, no combate a microorganismos resistentes. Portanto, a presença de metabólitos como taninos, esteróides e flavonóides estão associados à potente atividade analgésica em diferentes modelos de dor de origem neurogênica e inflamatória em camundongos, mesmo sendo compostos de natureza química diferente (TOLEDO et al., 2003).

Tabela 1. Resultados da prospecção fitoquímica de Tynnanthus fasciculatus MIERS em diferentes solventes. Frações preparadas a $10 \%$ (v/v - extrato seco/solvente) para todas as reações químicas. (A-I: reação formol clorídrico; A-II: gelatina salgada; B-I: reação da cianidina; B-II: reação com ácido sulfúrico concentrado; C-I: Baljet; C-II: Kedde; ${ }^{*}(+)$ resultado positivo e $(-)$ resultado negativo).

\begin{tabular}{|c|c|c|c|c|}
\hline Testes & & Áqua & Etanol & Diclorometano \\
\hline Açúcares Redutores & & $++++^{*}$ & +++ & --- \\
\hline Compostos Fenólicos & & +++ & +++ & --- \\
\hline \multirow[t]{2}{*}{ Taninos } & A-I & --- & +++ & +++ \\
\hline & A-II & +++ & +++ & --- \\
\hline \multirow[t]{2}{*}{ Flavonóides } & B- I & +++ & +++ & --- \\
\hline & B-II & +++ & +++ & +++ \\
\hline Cumarinas & & --- & --- & --- \\
\hline \multirow[t]{2}{*}{ Heterosídeos Cardiotônicos } & C- I & +++ & -- & +++ \\
\hline & C-II & +++ & -- & -- \\
\hline \multirow{2}{*}{$\begin{array}{l}\text { Saponinas } \\
\text { Triterpenos e Esteróides }\end{array}$} & & +++ & +++ & --- \\
\hline & & +++ & +++ & -- \\
\hline Naftoquinonas & & --- & --- & --- \\
\hline
\end{tabular}

MARTÍNEZ-FLORES (2002) relata que os flavonóides atuam na estabilização de espécies reativas de oxigênio por possuírem grupo de orto-dihidroxi ou grupo catecol no anel $\mathrm{B}$, o que confere maior estabilidade à forma radicalar. Por outro lado, as plantas são capazes de produzir diferentes substâncias tóxicas em grandes quantidades, como alguns alcalóides, cumarinas e outras provenientes do metabolismo secundário, aparentemente justificado para sua própria defesa contra vírus, bactérias, fungos e animais predadores. Entretanto, são poucos os estudos toxicológicos e genotóxicos (xenobiótico - danoso ao DNA) dessas substâncias (HAVSTEEN, 2002; ROSA et al., 2008).

Segundo SANCHES et al., (2005), várias espécies de Stryphnodendron contém cerca de $20 \%$ de taninos e são usadas na medicina popular como cicatrizantes, além de agirem como antimicrobianas e desativadoras de radicais livres, ou seja, antioxidantes. Tais espécies são derivados fenólicos, com uma boa solubilidade em água, de massa molecular entre 0,5 a $3 \mathrm{kDa}$. Muitas vezes, são os princípios ativos de plantas empregadas na medicina tradicional para o tratamento de diversas moléstias, por apresentarem atividades biológicas como a ação bactericida, fungicida, moluscicida e inibição enzimática, que podem ser potencialmente empregadas como novos princípios ativos e/ou outras aplicações (SILVA et al., 2004). 
Tabela 2. Relação dos metabólitos secundários encontrados na prospecção fitoquímica de Tynnanthus fasciculatus e suas possíveis atividades.

\begin{tabular}{c|ll}
\hline $\begin{array}{c}\text { Metabólitos } \\
\text { secundários }\end{array}$ & & Atividades e funções \\
\hline Compostos Fenólicos & - & Inibem a peroxidação lipídica e a lipooxigenase in vitro. \\
\hline Taninos & - & Ação antioxidante com potencial terapêutico no combate ao vitiligo, já que uma das \\
& causas desta doença é produção de radicais livres; & \\
& - & Anti-hipertensivo, antirreumático, antihemorrágico, cicatrizante, antiulcerogênico, e \\
& antiinflamatório em geral. \\
\hline Flavonóides & - & São pigmentos naturais, presentes nos vegetais; \\
& - & Proteção de plantas contra raios ultravioleta e visível; \\
& - & Atração de insetos com finalidade de polinização; \\
& - & Ação antioxidante; \\
& - & Controle da ação de hormônios vegetais; \\
& - & Alelopatia como marcadores taxonômicos. \\
\hline Heterosídeos & - & Tratamento de insuficiência cardíaca congestiva; \\
Cardiotônicos & - & Na profilaxia e tratamento de algumas arritmias como fibrilação atrial e taquicardia \\
& & atrial paroxística; \\
& - & Tratamento de choque cardiogênico, quando acompanhado de edema pulmonar. \\
\hline Saponinas & - & Antifúngica; \\
& - & Hipocolesterolimiantes; \\
\hline Triterpenos e & - & Espermicida. \\
Esteróides & - & Antiinflamatório e hormonal; \\
& - & Estrutura das células, transporte de elétrons, fotossíntese, sinalização celular, \\
\hline
\end{tabular}

(Fonte: SIMÕES et al., 2000)

A toxicidade da Artemia salina tem mostrado uma boa correlação com atividades antitumoral, inseticida e anti-Trypanosoma cruzi (ALVES et al., 2000) para substâncias que apresentam uma $\mathrm{DL}_{50}<10^{3} \mathrm{mg} / \mathrm{L}$ (MEYER et al., 1982; RUIZ et al., 2005).

A comunidade científica vem procurando compreender os fenômenos responsáveis pelos mecanismos adaptados de resistência a microorganismos, de forma criar alternativas e novas estratégias para o combate a bactérias resistentes. A demanda crescente por novas substâncias capazes de inibir, em concentrações baixas, processos vitais de uma ou mais espécies de microrganismos resistentes tem provocado uma verdadeira corrida em busca de agentes antimicrobianos cada vez mais eficientes, tanto de origem natural, quanto semi-sintéticos ou sintéticos (SILVEIRA et al., 2006).

Apesar dos mais otimistas já terem abandonado o antigo sonho de encontrar uma substância antibacteriana perfeita, ou seja, invencível frente à patógenos multiresistentes, com amplo espectro de ação, baixo e/ou sem efeitos adversos, o descobrimento de novos antibióticos potentes e mais seguros representa não apenas o avanço para uma melhor qualidade de vida, mas também a participação em um mercado em crescimento internacional que movimenta bilhões de dólares anualmente (SUTCLIFFE, 2003).

O teste de toxicidade frente à Artemia salina constitui um modelo de baixo custo e prático para análises de substâncias tóxicas devido a sua alta sensibilidade a quaisquer alterações do meio, o que permitiu verificar no decorrer do teste uma mortalidade crescente, sendo quase instantânea para concentrações, tanto mais elevadas do extrato, quanto para o controle positivo, podendo inferir que o uso de concentrações maiores que as adaptadas seriam letais para estripe de Artemia sp., ao passo que nas proporções menores não se verificou morte dos indivíduos.

O extrato aquoso do caule de Tynnanthus fasciculatus MIERS foi submetido ao bioensaio com Artemia sp., onde o mesmo proporcionou a relação de organismos (vivos e mortos) no final do ensaio, dispostos na Figura 2. Correlacionando os dados de porcentagem de morte versus concentração de extrato, a regressão linear entre esses dois parâmetros nos permite estimar uma $D_{50}=43,4 \mathrm{mg} \mathrm{L}^{-1}$ para o extrato. Esse valor indica uma alta toxicidade do extrato levando-se em consideração os dados fornecidos pela Organização Mundial de Saúde (OMS), que considera tóxica a substância que apresenta valores de $\mathrm{DL}_{50}<10^{3} \mathrm{mg} \mathrm{L}^{-1}$ frente à Artemia salina (MEYER et al., 1982). A maior atividade tóxica concentrou-se em torno de $100 \mathrm{mg} \mathrm{L}^{-1}$ onde houve a morte de $100 \%$ da população analisada.

A Tynnanthus fasciculatus MIERS está incluída numa das poucas espécies com atividade antifúngica conhecidas, ratificando a presença de metabólitos secundários identificados na prospecção fitoquímica. Isso foi também observado por MOREIRA et al., (2003) ao realizarem estudos de toxicidade frente à Artemia salina, onde foi verificado que a fração dos extratos analisados que continham triterpenos apresentaram uma maior ação citotóxica, podendo assim constituir uma barreira física na prevenção de ataques microbianos. 


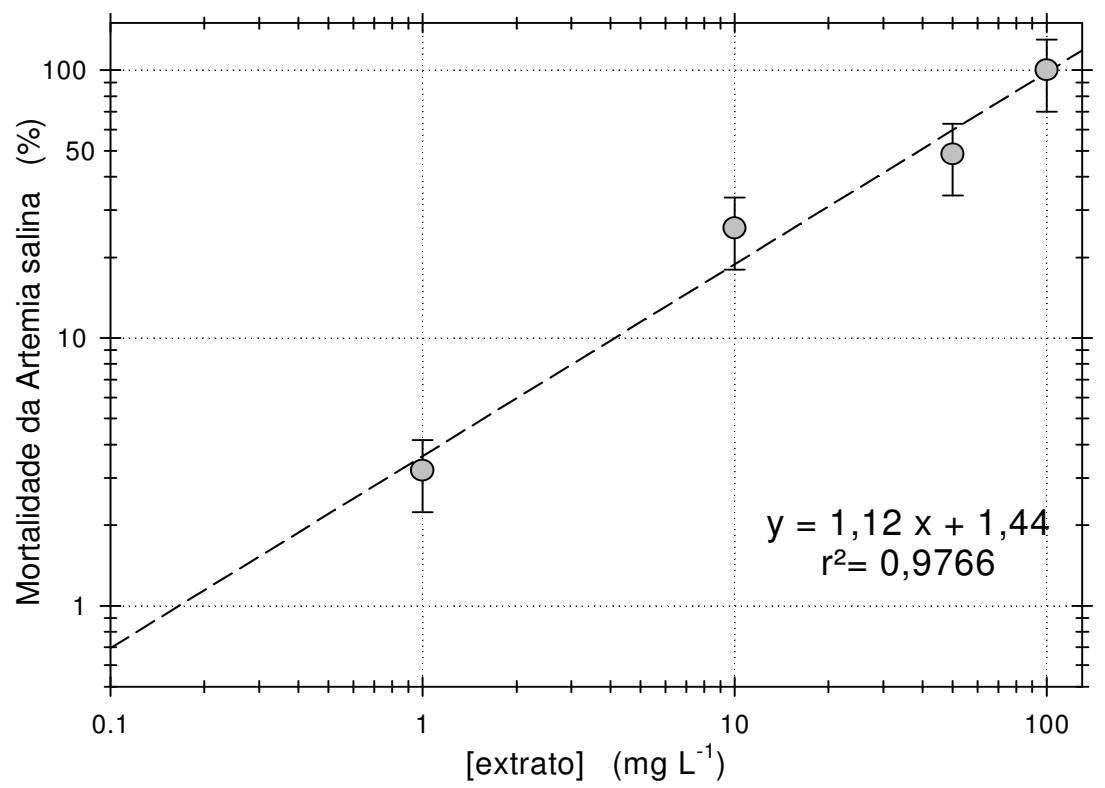

Figura 2. Porcentagem de larvas de Artemia mortas em relação às concentrações do extrato aquoso de Tynnanthus fasciculatus MIERS.

Tal fato indica uma correlação entre a presença destas classes de metabólitos secundários no extrato aquoso de Tynnanthus fasciculatus MIERS e a toxicidade observada frente à Artemia salina, ou seja, que esta atividade pode derivar ou ser causada pela presença dessas classes de compostos, onde a literatura tem mostrado que as atividades antioxidante e antifúngica podem estar relacionadas ao tipo de compostos fenólicos presentes em uma ampla variedade de vegetais fato que motivou a possibilidade de se associar a atividade antifúngica e antimicotoxigênica com a atividade antioxidante (OLIVEIRA et al., 2007).

Os compostos fenólicos possuem comprovada ação antifúngica e esta pode ocorrer, entre outros mecanismos, pela inativação de sistemas enzimáticos do microrganismo envolvidos na produção de energia e na síntese de componentes estruturais. A atividade antioxidante dos extratos vegetais, o que torna possível e a atividade da peroxidase pode indicar seu potencial de inibição do desenvolvimento fúngico e produção de micotoxinas (CHEN et al., 1998; VELIOGLU, 1998; OLIVEIRA et al., 2007).

\section{CONCLUSÕES}

O aparecimento de algumas doenças e o alto custo dos medicamentos tem levado a população a procurar o apoio na medicina alternativa, fazendo uso alternativo de plantas medicinais, por estes serem de fácil acesso e praticamente sem custo. Porém, o uso indiscriminado de certas plantas sem conhecermos o verdadeiro potencial tóxico, bem como a ação farmacológica de todas as espécies pode causar um efeito nocivo e não profilático desejado.

O estudo fitoquímico de Tynnanthus fasciculatus MIERS permitiu a averiguação de algumas classes de compostos globais constituintes em diferentes extratos da planta. Esta relação dos metabólitos secundários encontrados na prospecção fitoquímica permitiu correlacionar as suas possíveis atividades/funções, com as descritas por SIMÕES et al., (2000). Os resultados toxicológicos frente à Artemia sp. mostraram a necessidade de estudos posteriores mais específicos, principalmente tratando-se dos mecanismos de ação dos metabólitos em nível fisiológico, para que se possa correlacionar, por exemplo, com o efeito antifúngico e suas demais ações. Assim, torna-se possível caracterizar melhor o potencial e uso medicinal da planta in natura, visto que a espécie é tóxica em baixas concentrações e representa um risco para a população que a utiliza sem um prévio conhecimento.

\section{REFERÊNCIAS BIBLIOGRÁFICAS}

ABEL, P.D. Water Pollution Biology. Ellis Horwood Ltd, Publishers, Chichester, 1989.

ALVES T.M.D. Biological screening of Brazilian medicinal plants. Instituto Oswaldo Cruz v.95, p. 367-373, 2000. 
BALADRIN, M.F. et al.. Natural plant chemicals. Source of industrial and medicinal materials. Science, v.228, p.1054-1060, 1985.

BURGO, R. P.; N.K. ISHIKAWA; J. P. PINTO; FARIA, T. J.. Estudo da composição química e atividade antifúngica do óleo essencial de Tynnanthus fasciculatus. In: 29를 Reunião Anual da Sociedade Brasileira de Química, 2006.

CALOW, P.. Marine and Estuarine Invertebrate Toxicity Tests. In: Hoffman, D; Rattner,B; Burton,A \& Carns, J. Handbook in Citotoxicology. v.1. Blackwell Scientific Plubication, 1993.

CARVALHO et al.. Estudo espectrométrico de diferentes estágios fenológicos da Brassica oleracea var. capitata. Revista Brasileira de Farmacognosia, v.18, p.249-257, 2008.

CAVALCANTE, M.F. Síntese de 1,3,5-Triazinas substituídas e avaliação da toxicidade frente Artemia Salina. Química Nova, v. 23, n.1, 2000.

CHEN, Z. Y et al.. Antioxidative activity of green tea catechin extract compared with that of Rosemey extract. Journal Analytical Official Chemistry Society, v. 75, n. 9, p. 327-333, 1998.

COLEGATE, S. M.; MOLYNEUX, R. J. Bioactive Natural Products: Detection, Isolation and Structural Determination. CRC Press. London, 1993.

DI STASI, L.C. Plantas Medicinais: arte e ciência. São Paulo, SP: Unesp, 1996.

FENNER, R. et al. Plantas utilizadas na medicina popular brasileira com potencial atividade antifúngica. Revista Brasileira de Ciências Farmacêuticas. v. 42, n. 3, p. 369-394, 2006.

HAVSTEEN, B. H. The biochemistry and medical significance of the flavonóides. Pharmacology \& Therapeutics, v. 96, p. 67-202, 2002.

LORENZI, H. Árvores Brasileiras I, Instituto Plantarum de Estudos da Flora LTDA: Nova Odessa, 1988.

MARTÍNEZ-FLÓREZ, S. et al. Los flavonoides: propiedades y acciones antioxidantes. Nutrição Hospitalar. v. 17, n. 6, p.271-278, 2002.

MELO, F. C. S. A. Efeito da infusão do caule de cipó-cravo (Tynnanthus fasciculatus MIERS, Bignoniaceae) sobre as características morfométricas de componentes testiculares de ratos Wistar adultos. 2007. 87p. Tese (Mestrado) Programa de Pós-Graduação em Biologia celular e estrutural, Universidade Federal de Viçosa, Viçosa.

MEYER, B.N. et al. A convenient general bioassay for active plant constituents. Planta Medica, v.45, p.31-34, 1982.

MOREIRA, F. P. M. et al. Flavonóides e triterpenos de Baccharis pseudotenuifolia - Bioatividade sobre Artemia salina. Química Nova, v. 26, n. 3, p. 309-311, 2003.

OLIVEIRA, M. S. et al. Atividade antioxidante e antifúngica de extratos vegetais. Alimento e Nutrição. v.18, n.3, p. 267-275, 2007.

PAULETTI, P. M.; BOLZANI, V. S.; YOUNG, M. C. M. Constituintes Químicos de Arrabidaea Samydoides (Bignoniaceae). Química Nova. v. 26, n. 5, p.641-643, 2003.

ROSA, M. B. et al. Estudo espectrofotométrico da atividade fotoprotetora de extratos aquosos de Achillea millefolium, Brassica oleracea var. capitata, Cyperus rotundus, Plectranthus barbatus, Porophyllum ruderale (JACQ.) cass e Sonchus oleraceus. Revista Eletrônica de Farmácia. v.5, n.1, p.101-110, 2008.

RUIZ, A.L.T.G. et al. Avaliação da atividade tóxica em Artemia salina e Biomphalaria glabrata de extratos de quatro espécies do gênero Eleocharis (Cyperaceae). Revista Brasileira de Farmacognosia. v.15, n. 2, p.98-102, 2005.

SANCHES, A.C.C. et al. Antioxidant and antifungal activities of extracts and condensed tannins from Stryphnodendron obovatum Bent. Brazilian Journal of Pharmaceutical Sciences. v. 41, n. 1, p. 101-106, 2005. 
SILVA, H.H.G. et al. Atividade lavicida de taninos isolados de Magonia pubescens St. Hil. (Sapindaceae) sobre Aedes aegypti (Díptera, Culicidae). Revista da Sociedade Brasileira de Medicina Tropical. v. 37, n. 5, p. 396-399, 2004.

SILVEIRA, G. P. et al. Estratégias utilizadas no combate a resistência bacteriana. Química Nova. v. 29, n. 4, p. 844-855, 2006.

SIMÕES, C. O. et al. Farmacognosia da Planta ao Medicamento. Porto Alegre:Florianópolis: Editora UFRGS/ Editora UFSC, 1999.

SIQUEIRA, J.M. et al. Estudo fitoquímico de Unonopsis lindmanii - Annonaceae, biomonitorado pelo ensaio de toxicidade sobre Artemia Salina. Química Nova, v. 21, n. 5, p. 557-559, 1998.

SUTCLIFFE, J. A.; Antibacterial agents: solutions for the evolving problems of resistance. Bioorganic and Medicinal Chemistry Letters, v.13, n.23, p.4159- 4161, 2003.

TOLEDO, A. C. O. et al. Fitoterápicos: uma abordagem farmacotécnica. Revista Lecta, v. 21, n. 1, p.7-13, 2003.

VELIOGLU, Y. S. Antioxidant activity and total phenolic in selected fruits, vegetables, and grain products. Journal of Agricultural and Food Chemistry. v. 46, p. 4113-4117, 1998. 